\title{
Quadrupole Hyperfine Structure in the Rotational Spectra of Sulfuryl Chloride
}

\author{
Ilona Merke and Helmut Dreizler \\ Abteilung Chemische Physik im Institut für Physikalische Chemie der Universität Kiel, Kiel, FRG
}

Z. Naturforsch. 47a, 1153-1156 (1992); received September 12, 1992

\begin{abstract}
The nuclear quadrupole coupling of $\left[{ }^{35} \mathrm{Cl},{ }^{35} \mathrm{Cl}\right]$ - and $\left[{ }^{35} \mathrm{Cl},{ }^{37} \mathrm{Cl}\right]$-sulfuryl chloride, $\mathrm{SO}_{2} \mathrm{Cl}_{2}$ was investigated using waveguide microwave Fourier transform (MWFT) spectroscopy. The analysis was performed with direct diagonalization of the Hamiltonian matrix. It was possible to determine the complete coupling tensor with high accuracy. $\mathrm{SO}_{2}{ }^{35} \mathrm{Cl}_{2}: \chi_{a a}\left({ }^{35} \mathrm{Cl} 1\right)=\chi_{a a}\left({ }^{35} \mathrm{Cl} 2\right)=-33.049(6)$ $\mathrm{MHz}, \quad \chi_{c c}\left({ }^{35} \mathrm{Cl} 1\right)=\chi_{c c}\left({ }^{35} \mathrm{Cl} 2\right)=40.356(9) \mathrm{MHz}, \quad \chi_{a b}\left({ }^{35} \mathrm{Cl} 1\right)=-\chi_{a b}\left({ }^{35} \mathrm{Cl} 2\right)= \pm 52.67(63) \mathrm{MHz}$. $\mathrm{SO}_{2}{ }^{35} \mathrm{Cl}^{37} \mathrm{Cl}: \chi_{a a}\left({ }^{35} \mathrm{Cl}\right)=-29.582(17) \mathrm{MHz}, \chi_{c c}\left({ }^{35} \mathrm{Cl}\right)=40.340(25) \mathrm{MHz}, \quad \chi_{a b}\left({ }^{35} \mathrm{Cl}\right)= \pm 52.3(14)$ $\mathrm{MHz}, \chi_{a a}\left({ }^{37} \mathrm{Cl}\right)=-28.723(18) \mathrm{MHz}, \chi_{c c}\left({ }^{37} \mathrm{Cl}\right) \stackrel{\chi_{c c}}{=} 31.822(28) \mathrm{MHz}, \chi_{a b}\left({ }^{37} \mathrm{Cl}\right)=\frac{\chi_{a b}}{+} 37.3(19) \mathrm{MHz}$.

We also determined the quadrupole coupling constants in their principal axes system. In comparison with the structure, the $z$-axis of the coupling tensor was found to be tilted $2.0^{\circ}$ out off the $\mathrm{S}-\mathrm{Cl}$ bond axis.
\end{abstract}

\section{Introduction}

The microwave spectrum of $\mathrm{SO}_{2} \mathrm{Cl}_{2}$ was measured for the first time by Abbar [1] in 1963. In two following papers $[2,3]$ he supplemented his work. In [2] he also reported on the analysis of the ${ }^{35} \mathrm{Cl}$ nuclear quadrupole coupling in $\mathrm{SO}_{2}{ }^{35} \mathrm{Cl}_{2}$. Since the spectral resolution was 100 to $200 \mathrm{kHz}$, the coupling constants had a rather large standard error of $1 \mathrm{MHz}$. Burie et al. [4] and Debrulle et al. [5] investigated the centrifugal distortion effects of $\mathrm{SO}_{2}{ }^{35} \mathrm{Cl}_{2}$. The assignment of the rotational spectrum of $\mathrm{SO}_{2}{ }^{35} \mathrm{Cl}^{37} \mathrm{Cl}$ was reported in $[6,7]$.

In this work we investigated the spectra of $\mathrm{SO}_{2}{ }^{35} \mathrm{Cl}_{2}$ and $\mathrm{SO}_{2}{ }^{35} \mathrm{Cl}^{37} \mathrm{Cl}$ with the high resolution of microwave Fourier transform (MWFT) spectroscopy [8]. It was possible to improve the accuracy of the diagonal elements and to determine the off diagonal elements of the quadrupole coupling tensor of $\mathrm{SO}_{2}{ }^{35} \mathrm{Cl}_{2}$. Additionally we determined the quadrupole coupling of $\mathrm{SO}_{2}{ }^{35} \mathrm{Cl}^{37} \mathrm{Cl}$ for the first time [9].

\section{Experimental}

All measurements were performed with waveguide MWFT-spectrometers [10-12] in the frequency range

Reprint requests to Prof. Dr. H. Dreizler, Abteilung Chemische Physik, Institut für Physikalische Chemie, Universität Kiel, Olshausenstr. 40-60, W-2300 Kiel 1, FRG. from 6 to $26.5 \mathrm{GHz}$, at a pressure of $0.3 \mathrm{~Pa}(2 \mathrm{mTorr})$ and temperatures around $233 \mathrm{~K}\left(-40^{\circ} \mathrm{C}\right)$. In the spectrum of $\mathrm{SO}_{2} \mathrm{Cl}_{2}$ the required transitions were often superimposed by strong perturbing transitions. We assume these to be high $J$ transitions or transitions belonging to vibrationally excited states. Also lines of $\mathrm{SO}_{2}$ were detected. In order to measure these superimposed transitions, double resonance experiments [13] were carried out.

Some selected frequencies are presented in Tables 1 and 2. A complete list is given in [9] and deposited under TNA 29 at the library of the University, D-2300 Kiel, Westring 400. In Fig. 1 we show an example for a transition without and with the double resonance technique. To eliminate overlap effects [14] the frequencies were determined by a least squares fit of the time domain signal [15].

\section{Analysis and Discussion}

The analysis of the hyperfine structure was analyzed with the program Q2DIAG described in [16]. The Hamiltonian matrix was set up in a coupled basis. The coupling scheme $I_{1}+I_{2}=I, I+J=F$ was used. The Hamiltonian is based on considerations in [17]. We used $\chi_{a a}, \chi_{b b}, \chi_{a b}$ and the hypothetical unsplit frequencies as fit parameters. The rotational constants for $\mathrm{SO}_{2}{ }^{35} \mathrm{Cl}_{2}$ were fixed at the values $A=3485.92$ $\mathrm{MHz}, B=2344.31 \mathrm{MHz}, C=1930.37 \mathrm{MHz}$ given in [5]. For $\mathrm{SO}_{2}{ }^{35} \mathrm{Cl}^{37} \mathrm{Cl}$ the rotational constants $A=$

0932-0784 / $92 / 1100-1153 \$ 01.30 / 0$. - Please order a reprint rather than making your own copy. 
Table 1. Selected rotational transitions of sulfuryl chloride $\mathrm{SO}_{2}{ }^{35} \mathrm{Cl}_{2}$ in the vibrational ground state. $v$ : measured frequency [MHz], $\delta_{\mathrm{hfs}}$ : observed-minus-calculated frequency of the hyperfine component $[\mathrm{kHz}], v_{0}$ : hypothetical center frequency $[\mathrm{MHz}]$.

\begin{tabular}{|c|c|c|c|c|c|c|c|c|c|c|c|c|c|}
\hline \multicolumn{7}{|c|}{ he hyperfine component $[\mathrm{kHz}], v_{0}$ : hypothetical } & \multirow{2}{*}{ 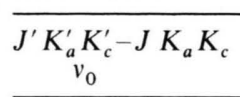 } & & & & & & \\
\hline 16 ing & & & & & & & & $I^{\prime}$ & $F^{\prime}$ & $I$ & $F$ & $v$ & $\delta_{\mathrm{hfs}}$ \\
\hline $\begin{array}{c}J^{\prime} K_{a}^{\prime} K_{c}^{\prime}-J K_{a} K_{c} \\
v_{0} \\
\end{array}$ & $I^{\prime}$ & $F^{\prime}$ & $I$ & $F$ & $v$ & $\delta_{\mathrm{hfs}}$ & \multirow{2}{*}{$\begin{array}{l}1111-0 \quad 0 \quad 0 \\
5347.4475(80)\end{array}$} & $\begin{array}{l}3 \\
2\end{array}$ & $\begin{array}{l}2 \\
1 \\
1\end{array}$ & $\begin{array}{l}3 \\
2 \\
1\end{array}$ & $\begin{array}{l}3 \\
2 \\
1\end{array}$ & $\begin{array}{l}5350.418 \\
5350.347 \\
5348.545\end{array}$ & \multirow[b]{2}{*}{$\begin{array}{r}-9.5 \\
-0.0 \\
-6.4 \\
-5.1 \\
0.4 \\
-1.7 \\
-6.1 \\
9.7\end{array}$} \\
\hline $\begin{array}{llllll}1 & 1 & 1 & -0 & 0 & 0 \\
5416.1566(56)\end{array}$ & $\begin{array}{l}2 \\
2 \\
2 \\
2 \\
0 \\
0\end{array}$ & $\begin{array}{l}1 \\
1 \\
2 \\
3 \\
1 \\
1\end{array}$ & $\begin{array}{l}2 \\
0 \\
2 \\
2 \\
2 \\
0\end{array}$ & $\begin{array}{l}2 \\
0 \\
2 \\
2 \\
2 \\
0\end{array}$ & $\begin{array}{lll}5 & 418.632 \\
5 & 418.591 \\
5 & 416.494 \\
5 & 416.284 \\
5 & 413.968 \\
5 & 413.924\end{array}$ & $\begin{array}{r}22.5 \\
11.8 \\
3.9 \\
4.6 \\
-14.4 \\
-28.1\end{array}$ & & $\begin{array}{l}2 \\
1 \\
3 \\
2 \\
2 \\
1 \\
3\end{array}$ & $\begin{array}{l}1 \\
1 \\
4 \\
2 \\
3 \\
2 \\
4\end{array}$ & $\begin{array}{l}2 \\
1 \\
3 \\
2 \\
2 \\
1 \\
3\end{array}$ & $\begin{array}{l}2 \\
1 \\
3 \\
2 \\
2 \\
1 \\
4\end{array}$ & $\begin{array}{l}5350.347 \\
5348.545 \\
5348.138 \\
5347.980 \\
5347.779 \\
5345.799 \\
5386.528\end{array}$ & \\
\hline $\begin{array}{llllll}2 & 0 & 2 & -1 & 1 & 1 \\
7313 & 81 & 18(56)\end{array}$ & $\begin{array}{l}2 \\
2 \\
2 \\
2 \\
0 \\
0\end{array}$ & $\begin{array}{l}2 \\
2 \\
4 \\
3 \\
2 \\
2\end{array}$ & $\begin{array}{l}2 \\
2 \\
2 \\
2 \\
0 \\
2\end{array}$ & $\begin{array}{l}2 \\
1 \\
3 \\
2 \\
1 \\
2\end{array}$ & $\begin{array}{ll}7 & 323.928 \\
7 & 321.839 \\
7 & 313.711 \\
7 & 313.546 \\
7 & 305.594 \\
7 & 303.132\end{array}$ & $\begin{array}{r}-26.4 \\
4.0 \\
6.2 \\
6.2 \\
-17.7 \\
28.0\end{array}$ & $5372.9398(26)$ & $\begin{array}{l}0 \\
1 \\
3 \\
3 \\
1 \\
2 \\
2\end{array}$ & $\begin{array}{l}3 \\
2 \\
5 \\
3 \\
4 \\
5 \\
4\end{array}$ & $\begin{array}{l}0 \\
1 \\
3 \\
3 \\
2 \\
2 \\
1\end{array}$ & $\begin{array}{l}3 \\
2 \\
5 \\
3 \\
4 \\
5 \\
4\end{array}$ & $\begin{array}{l}5384.545 \\
5381.089 \\
5380.580 \\
5378.623 \\
5373.144 \\
5372.767 \\
5371.057\end{array}$ & $\begin{array}{r}-9.0 \\
3.1 \\
1.5 \\
-11.7 \\
-3.3 \\
-1.0 \\
-3.8\end{array}$ \\
\hline $\begin{array}{lllll}3 & 1 & 3-2 & 0 & 2 \\
12 & 970.9027 & (39)\end{array}$ & $\begin{array}{l}2 \\
2 \\
2 \\
0\end{array}$ & $\begin{array}{l}3 \\
5 \\
4 \\
3\end{array}$ & $\begin{array}{l}2 \\
2 \\
2 \\
0\end{array}$ & $\begin{array}{l}2 \\
4 \\
3 \\
2\end{array}$ & $\begin{array}{l}12973.462 \\
12970.943 \\
12970.910 \\
12968.360\end{array}$ & $\begin{array}{r}-5.5 \\
6.8 \\
4.9 \\
-6.0\end{array}$ & & $\begin{array}{l}3 \\
3 \\
2 \\
1 \\
3\end{array}$ & $\begin{array}{l}1 \\
6 \\
3 \\
3 \\
2\end{array}$ & $\begin{array}{l}3 \\
3 \\
2 \\
1 \\
3\end{array}$ & $\begin{array}{l}2 \\
6 \\
3 \\
3 \\
1\end{array}$ & $\begin{array}{l}5366.032 \\
5365.472 \\
5361.852 \\
5360.743 \\
5359.446\end{array}$ & $\begin{array}{r}-11.9 \\
2.6 \\
5.3 \\
16.7 \\
12.6\end{array}$ \\
\hline $\begin{array}{llllll}4 & 0 & 4 & -3 & 1 & 3 \\
15 & 86 & 62.5654 & (44)\end{array}$ & $\begin{array}{l}0 \\
2 \\
2\end{array}$ & $\begin{array}{l}4 \\
6 \\
4\end{array}$ & $\begin{array}{l}2 \\
2 \\
0\end{array}$ & $\begin{array}{l}3 \\
5 \\
3\end{array}$ & $\begin{array}{l}15863.143 \\
15862.569 \\
15861.986\end{array}$ & $\begin{array}{r}-3.9 \\
2.5 \\
1.5\end{array}$ & 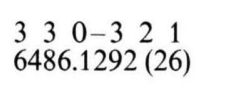 & $\begin{array}{l}3 \\
3 \\
0 \\
1\end{array}$ & $\begin{array}{l}2 \\
4 \\
3 \\
2\end{array}$ & $\begin{array}{l}3 \\
3 \\
0 \\
1\end{array}$ & $\begin{array}{l}1 \\
4 \\
3 \\
2\end{array}$ & $\begin{array}{l}5359.446 \\
6494.181 \\
6492.939 \\
6490.864\end{array}$ & $\begin{array}{r}12.6 \\
7.3 \\
-4.4 \\
-3.7\end{array}$ \\
\hline $\begin{array}{llllll}4 & 1 & 4 & -3 & 0 & 3 \\
16 & 610.9724 & (26)\end{array}$ & $\begin{array}{l}3 \\
1 \\
3 \\
1 \\
1 \\
3 \\
3 \\
3 \\
3\end{array}$ & $\begin{array}{l}7 \\
4 \\
6 \\
5 \\
3 \\
2 \\
3 \\
5 \\
4\end{array}$ & $\begin{array}{l}3 \\
1 \\
3 \\
1 \\
1 \\
3 \\
3 \\
3 \\
3\end{array}$ & $\begin{array}{l}6 \\
3 \\
5 \\
4 \\
2 \\
1 \\
2 \\
4 \\
3\end{array}$ & $\begin{array}{l}16613.349 \\
16613.062 \\
16611.146 \\
16610.583 \\
16609.320 \\
16608.935 \\
16608.421 \\
16608.226 \\
16607.023\end{array}$ & $\begin{array}{r}0.7 \\
-8.3 \\
3.2 \\
4.0 \\
3.7 \\
-9.9 \\
2.2 \\
4.1 \\
0.3\end{array}$ & & $\begin{array}{l}3 \\
3 \\
3 \\
3 \\
2 \\
2 \\
2 \\
1 \\
3 \\
1\end{array}$ & $\begin{array}{l}2 \\
5 \\
3 \\
5 \\
3 \\
5 \\
1 \\
4 \\
4 \\
6 \\
3\end{array}$ & $\begin{array}{l}3 \\
3 \\
3 \\
3 \\
3 \\
2 \\
2 \\
1 \\
2 \\
3 \\
1\end{array}$ & $\begin{array}{l}5 \\
3 \\
4 \\
4 \\
5 \\
1 \\
4 \\
4 \\
6 \\
3\end{array}$ & $\begin{array}{l}6490.412 \\
6489.540 \\
6488.940 \\
6487.409 \\
6486.320 \\
6486.071 \\
6485.962 \\
6485.341 \\
6481.701 \\
6479459\end{array}$ & $\begin{array}{r}1.3 \\
2.4 \\
3.1 \\
-3.7 \\
0.7 \\
-5.7 \\
1.2 \\
-12.3 \\
-2.1 \\
18.6\end{array}$ \\
\hline 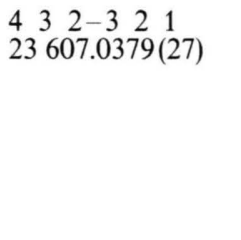 & $\begin{array}{l}3 \\
3 \\
3 \\
1 \\
1 \\
3 \\
1 \\
3\end{array}$ & $\begin{array}{l}4 \\
3 \\
5 \\
3 \\
5 \\
6 \\
4 \\
7\end{array}$ & $\begin{array}{l}3 \\
3 \\
3 \\
1 \\
1 \\
3 \\
1 \\
3\end{array}$ & $\begin{array}{l}3 \\
2 \\
4 \\
2 \\
4 \\
5 \\
3 \\
6\end{array}$ & $\begin{array}{ll}23 & 608.039 \\
23 & 607.852 \\
23 & 607.597 \\
23 & 607.374 \\
23 & 607.176 \\
23 & 606.904 \\
23 & 606.632 \\
23 & 606.498\end{array}$ & $\begin{array}{r}1.2 \\
-1.8 \\
5.9 \\
-3.7 \\
-4.4 \\
5.6 \\
-2.5 \\
0.2\end{array}$ & $\begin{array}{llllll}3 & 3 & 1 & -3 & 2 & 2 \\
6892 & 62 & 12 & (27)\end{array}$ & $\begin{array}{l}1 \\
2 \\
3 \\
0 \\
1 \\
3 \\
3 \\
2 \\
1\end{array}$ & $\begin{array}{l}3 \\
3 \\
4 \\
3 \\
2 \\
5 \\
5 \\
3 \\
5 \\
4\end{array}$ & $\begin{array}{l}1 \\
2 \\
3 \\
0 \\
1 \\
3 \\
3 \\
2 \\
2\end{array}$ & $\begin{array}{l}3 \\
3 \\
4 \\
3 \\
2 \\
5 \\
3 \\
5 \\
4\end{array}$ & $\begin{array}{l}6479.086 \\
6904.134 \\
6902.425 \\
6899.476 \\
6898.951 \\
6897.444 \\
6892.635 \\
6891.225\end{array}$ & $\begin{array}{r}10.0 \\
-6.0 \\
-6.9 \\
12.1 \\
-6.6 \\
-6.8 \\
-9.3 \\
-1.3 \\
3.0\end{array}$ \\
\hline $\begin{array}{llllll}4 & 2 & 3 & -4 & 1 & 4 \\
6248.1578 & (19)\end{array}$ & $\begin{array}{l}3 \\
3 \\
3 \\
1 \\
3 \\
3 \\
1 \\
3 \\
3 \\
3 \\
3 \\
3 \\
1 \\
3 \\
3 \\
3\end{array}$ & $\begin{array}{l}5 \\
3 \\
7 \\
3 \\
4 \\
6 \\
5 \\
2 \\
3 \\
4 \\
7 \\
2 \\
4 \\
3 \\
6 \\
1\end{array}$ & $\begin{array}{l}3 \\
3 \\
3 \\
1 \\
3 \\
3 \\
1 \\
3 \\
3 \\
3 \\
3 \\
3 \\
1 \\
3 \\
3 \\
3\end{array}$ & $\begin{array}{l}5 \\
4 \\
6 \\
3 \\
4 \\
6 \\
5 \\
3 \\
3 \\
3 \\
7 \\
2 \\
4 \\
2 \\
7 \\
1\end{array}$ & $\begin{array}{ll}6 & 260.548 \\
6 & 259.186 \\
6 & 258.403 \\
6 & 255.546 \\
6 & 255.426 \\
6 & 253.751 \\
6 & 247.180 \\
6 & 247.019 \\
6 & 245.022 \\
6 & 241.276 \\
6 & 240.704 \\
6 & 239.383 \\
6 & 237.680 \\
6 & 237.371 \\
6 & 236.048 \\
6 & 233.517\end{array}$ & $\begin{array}{r}-9.3 \\
13.6 \\
4.6 \\
-8.9 \\
-8.8 \\
0.5 \\
-5.5 \\
-10.3 \\
11.4 \\
3.0 \\
-0.2 \\
5.5 \\
1.7 \\
12.1 \\
-8.4 \\
-0.8\end{array}$ & $\begin{array}{llllll}4 & 2 & 3 & -4 & 1 & 4 \\
6260.7617 & (27)\end{array}$ & $\begin{array}{l}3 \\
3 \\
2 \\
1 \\
3 \\
3 \\
2 \\
1 \\
3 \\
3 \\
2 \\
2 \\
2 \\
1 \\
3 \\
0 \\
1\end{array}$ & $\begin{array}{l}2 \\
6 \\
3 \\
3 \\
1 \\
5 \\
4 \\
3 \\
4 \\
6 \\
3 \\
5 \\
6 \\
5 \\
7 \\
4 \\
4\end{array}$ & $\begin{array}{l}3 \\
3 \\
2 \\
1 \\
3 \\
3 \\
0 \\
1 \\
3 \\
3 \\
2 \\
2 \\
2 \\
1 \\
3 \\
2 \\
1\end{array}$ & $\begin{array}{l}1 \\
6 \\
3 \\
3 \\
2 \\
5 \\
4 \\
3 \\
4 \\
6 \\
3 \\
5 \\
6 \\
5 \\
7 \\
4 \\
4\end{array}$ & $\begin{array}{l}6887.054 \\
6886.304 \\
6882.900 \\
6882.685 \\
6881.262 \\
6271.853 \\
6270.145 \\
6267.414 \\
6267.240 \\
6265.973 \\
6261.275 \\
6261.177 \\
6260.548 \\
6259.455 \\
6254.095 \\
6251.821 \\
6250.983\end{array}$ & $\begin{array}{r}12.3 \\
-3.0 \\
-6.5 \\
0.2 \\
9.4 \\
-0.7 \\
1.6 \\
5.5 \\
5.3 \\
-0.8 \\
-8.6 \\
-3.3 \\
7.1 \\
-3.3 \\
-3.3 \\
6.6 \\
-5.3\end{array}$ \\
\hline 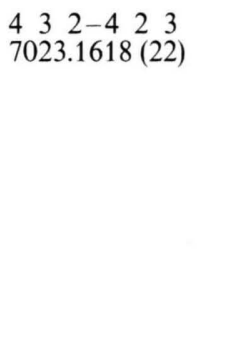 & $\begin{array}{l}3 \\
1 \\
3 \\
3 \\
1 \\
3 \\
3 \\
3 \\
3 \\
3 \\
1 \\
3\end{array}$ & $\begin{array}{l}5 \\
3 \\
4 \\
6 \\
5 \\
3 \\
3 \\
2 \\
7 \\
2 \\
4 \\
1\end{array}$ & $\begin{array}{l}3 \\
1 \\
3 \\
3 \\
1 \\
3 \\
3 \\
3 \\
3 \\
3 \\
1 \\
3\end{array}$ & $\begin{array}{l}5 \\
3 \\
4 \\
6 \\
5 \\
3 \\
2 \\
3 \\
7 \\
2 \\
4 \\
1\end{array}$ & $\begin{array}{ll}7 & 032.205 \\
7 & 028.576 \\
7 & 028.471 \\
7 & 027.254 \\
7 & 022.462 \\
7 & 020.874 \\
7 & 018.849 \\
7 & 018.764 \\
7 & 017.729 \\
7 & 016.743 \\
7 & 015.513 \\
7 & 012.474\end{array}$ & $\begin{array}{r}1.6 \\
10.6 \\
-5.6 \\
4.8 \\
-2.3 \\
1.2 \\
-5.1 \\
-9.1 \\
2.8 \\
-11.4 \\
1.7 \\
10.6\end{array}$ & 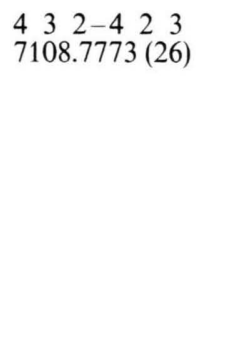 & $\begin{array}{l}3 \\
2 \\
2 \\
1 \\
3 \\
3 \\
2 \\
2 \\
1 \\
3 \\
3 \\
0 \\
1\end{array}$ & $\begin{array}{l}4 \\
5 \\
4 \\
3 \\
4 \\
6 \\
5 \\
6 \\
5 \\
3 \\
7 \\
4 \\
4\end{array}$ & $\begin{array}{l}3 \\
3 \\
2 \\
1 \\
3 \\
3 \\
2 \\
2 \\
1 \\
3 \\
3 \\
0 \\
1\end{array}$ & $\begin{array}{l}4 \\
5 \\
4 \\
3 \\
4 \\
6 \\
5 \\
6 \\
5 \\
3 \\
7 \\
4 \\
4\end{array}$ & $\begin{array}{l}7116.759 \\
7115.506 \\
7113.554 \\
7113.465 \\
7112.415 \\
7108.856 \\
7108.747 \\
7108.090 \\
7106.672 \\
7103.975 \\
7102.145 \\
7101.912\end{array}$ & $\begin{array}{r}-2.1 \\
-1.8 \\
1.6 \\
0.4 \\
1.1 \\
6.0 \\
-3.1 \\
1.8 \\
0.8 \\
-3.9 \\
-7.0 \\
-5.2\end{array}$ \\
\hline
\end{tabular}

Table 2. Selected rotational transitions of sulfuryl chloride $\mathrm{SO}_{2}{ }^{35} \mathrm{Cl}^{37} \mathrm{Cl}$ in the vibrational ground state. List of symbols see Table 1 . 
a)

b)

c)

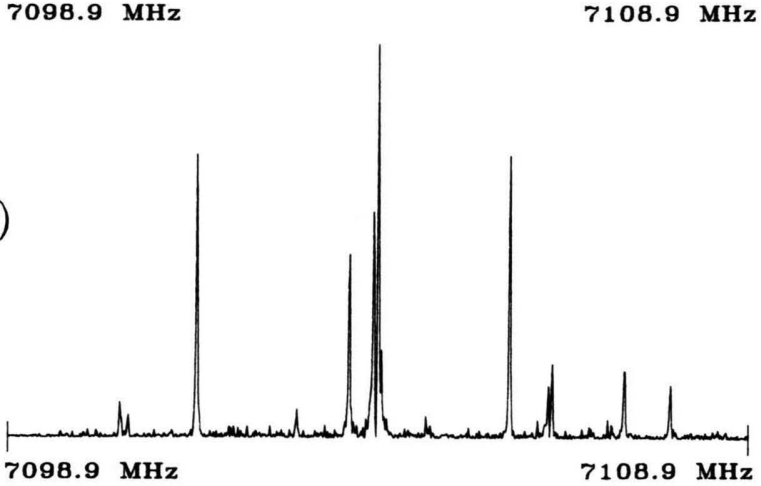

\subsection{MHz}

Fig. 1. Example of application of double resonance spectroscopy at the transition $J^{\prime} K_{a}^{\prime} K_{c}^{\prime}-J K_{a} K_{c}=4_{32}-4_{23}$ of $\mathrm{SO}_{2}{ }^{35} \mathrm{Cl}^{37} \mathrm{Cl}$. a) Without double resonance, recording conditions: pressure: $0.4 \mathrm{~Pa}(3.0 \mathrm{mTorr})$, polarization frequency: $7110.0 \mathrm{MHz}$, sample interval: $10 \mathrm{~ns}$, averaging cycles: $3.3 \cdot 10^{6}$, 1024 data points supplemented with 3072 zeros prior to Fourier transformation. b) Double resonance with $5_{23}-4_{32}$ at $15874.0 \mathrm{MHz}$. Pump power approximately $50 \mathrm{~mW}$, averaging cycles: $3.3 \cdot 10^{7}$, all other recording conditions were equivalent to a). c) Theoretical spectrum. The correct frequency on the right hand side is $7118.9 \mathrm{MHz}$ not $7108.9 \mathrm{MHz}$ in a) - c).

3459.39 MHz $, \quad B=2293.83 \mathrm{MHz}, \quad C=1888.16 \mathrm{MHz}$ given in [7] were used.

The axis perpendicular to the $\mathrm{Cl}-\mathrm{S}-\mathrm{Cl}$ plane is a principal axis of both the inertia and the coupling tensor ( $c$ - and $y$-axis, respectively). Therefore the knowledge of $\chi_{a b}$ allows the calculation of the angle

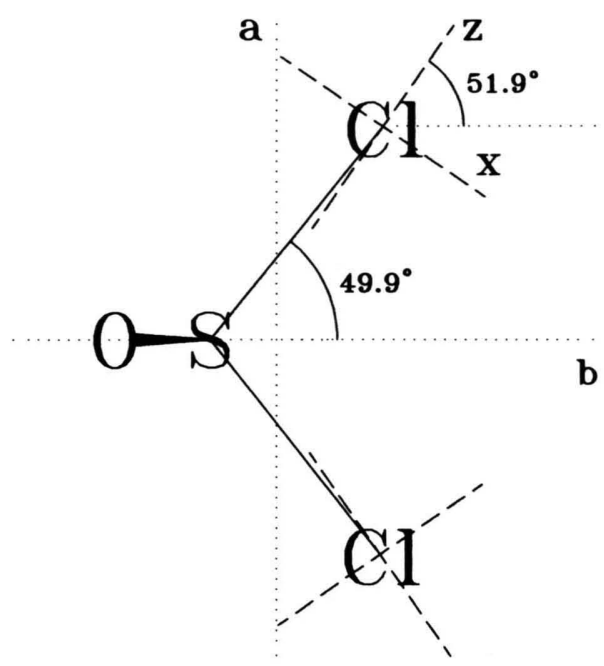

Fig. 2. Sulfuryl chloride in its principal inertia axes system $(a, b)$. The $x$ - and $z$-axes are principal axes of the quadrupole coupling tensor. The $z$-axis is tilted out of the $\mathrm{S}-\mathrm{Cl}$ internuclear axis by $2.0^{\circ}$. For better recognition this angle is drawn too large in the figure.

Table 3. Quadrupole coupling constants in $[\mathrm{MHz}]$ of ${ }^{35} \mathrm{Cl}^{35} \mathrm{Cl}$ and ${ }^{35} \mathrm{Cl}^{37} \mathrm{Cl}$-sulfuryl chloride. $\chi_{g g^{\prime}}\left(g, g^{\prime}=a, b, c\right)$ coupling constants in the principal inertia axes system. $\chi_{g g^{\prime}}\left(g, g^{\prime}=\right.$ $x, y, z)$ constants in the principal axes system of the coupling tensor. $\theta_{z a}$ : angle between the $a$-principal inertial axis and the $z$-principal axis of the coupling tensor in degrees. $q: \chi_{y y}\left({ }^{35} \mathrm{Cl}\right) /$ $\chi_{y y}\left({ }^{37} \mathrm{Cl}\right)$. Single standard error in brackets. $N$ : number of components included in the fit, $\sigma$ : standard deviation of the fit in $\mathrm{kHz}$.

\begin{tabular}{ll}
\hline $\mathrm{SO}_{2}{ }^{35} \mathrm{Cl}{ }_{2}$ & $\mathrm{SO}_{2}{ }^{37} \mathrm{Cl}{ }^{37} \mathrm{Cl}$ \\
\hline$\chi_{a a}\left({ }^{35} \mathrm{Cl} 1\right)=\chi_{a a}\left({ }^{35} \mathrm{Cl} 2\right)=-33.049(6)$ & $\chi_{a a}\left({ }^{35} \mathrm{Cl} 1\right)=-29.582(17)$ \\
$\chi_{b b}\left({ }^{35} \mathrm{Cl} 1\right)=\chi_{b b}\left({ }^{35} \mathrm{Cl} 2\right)=-7.307(9)$ & $\chi_{b b}\left({ }^{35} \mathrm{Cl} 1\right)=-10.758(25)$ \\
$\chi_{c c}\left({ }^{35} \mathrm{Cl} 1\right)=\chi_{c c}\left({ }^{35} \mathrm{Cl} 2\right)=40.356(9)$ & $\chi_{c c}\left({ }^{35} \mathrm{Cl} 1\right)=40.340(25)$ \\
$\chi_{a b}\left({ }^{35} \mathrm{Cl} 1\right)=-\chi_{a b}\left({ }^{35} \mathrm{Cl} 2\right)= \pm 52.67(63)$ & $\chi_{a b}\left({ }^{35} \mathrm{Cl} 1\right)= \pm 52.3(14)$ \\
& $\chi_{a a}\left({ }^{37} \mathrm{Cl} 1\right)=-28.723(18)$ \\
& $\chi_{b b}\left({ }^{37} \mathrm{Cl} 1\right)=-3.099(28)$ \\
& $\chi_{c c}\left({ }^{37} \mathrm{Cl} 1\right)=31.822(28)$ \\
$\theta_{z a}= \pm 38.136(79)$ & $\chi_{a b}\left({ }^{37} \mathrm{Cl} 1\right)=\mp 37.3(19)$ \\
& $\theta_{z a}\left({ }^{35} \mathrm{Cl}\right)= \pm 39.90(13)$ \\
$\chi_{x x}\left({ }^{35} \mathrm{Cl} 1\right)=\chi_{x x}\left({ }^{35} \mathrm{Cl} 2\right)=34.04(61)$ & $\theta_{z a}\left({ }^{37} \mathrm{Cl}\right)=\mp 35.52(45)$ \\
$\chi_{y y}\left({ }^{35} \mathrm{Cl} 1\right)=\chi_{y y}\left({ }^{35} \mathrm{Cl} 2\right)=40.3560(94)$ & $\chi_{x x}\left({ }^{35} \mathrm{Cl} 1\right)=33.0(14)$ \\
$\chi_{z z}\left({ }^{35} \mathrm{Cl} 1\right)=\chi_{z z}\left({ }^{35} \mathrm{Cl} 2\right)=-74.40(61)$ & $\chi_{z z}\left({ }^{35} \mathrm{Cl} 1\right)=40.340(25)$ \\
& $\chi_{x x}\left({ }^{37} \mathrm{Cl} 1\right)=-73.3(14)$ \\
& $\chi_{y y}\left({ }^{37} \mathrm{Cl} 1\right)=31.822(28)$ \\
& $\chi_{z z}\left({ }^{37} \mathrm{Cl} 1\right)=-55.4(18)$ \\
& $q=1.2677(89)$ \\
$N=142$ & $N=126$ \\
$\sigma={ }^{35} 7.7$ & $\left.\sigma={ }^{35}\right)$ \\
\hline
\end{tabular}


$\theta_{z a}$ between the $z$-principal axis of the coupling tensor and the $a$-principal axis of the inertia tensor as well as the $\chi_{q q}(q=x, y, z)$ elements in the principal axes system of the coupling tensor. For the axes systems see Figure 2. The electronic surrounding of the chlorine nuclei in both isotopomeres can be expected to be the same. Therefore we also expect the same coupling constants $\chi_{q q}(q=x, y, z)$ in the principal axes system of the coupling tensor of ${ }^{35} \mathrm{Cl}$ in $\mathrm{SO}_{2}{ }^{35} \mathrm{Cl}_{2}$ and $\mathrm{SO}_{2}{ }^{35} \mathrm{Cl}^{37} \mathrm{Cl}$. The experimental results are given in Table 3. A comparison with the structure [7] indicates that the $z$-axis of the coupling tensor is tilted by $2.0^{\circ}$ with respect to the $\mathrm{S}-\mathrm{Cl}$ bond axis. This supports the hypothesis of a bent bond between the sulfur and the chlorine nuclei.

Comparison of the sulfur chlorine compounds sulfur dichloride $\mathrm{SCl}_{2}$ [18], thionyl chloride, $\mathrm{SOCl}_{2}$ [19], and sulfuryl chloride, $\mathrm{SO}_{2} \mathrm{Cl}_{2}$, all of them measured with high resolution in our laboratory, is not strictly possible, as in the case of $\mathrm{SOCl}_{2}$ it was not possible to determine the three off diagonal elements $\chi_{g g^{\prime}}, g \neq g^{\prime}$ of the coupling tensor. The present limitation is by computer core and time. From the results it is obvious that the substitution of two oxygen atoms at the sulfur

1] C. Abbar, C. R. Acad. Sci. Paris 257, 2222 (1963).

[2] C. Abbar, C. R. Acad. Sci. Paris 261, 365 (1965).

[3] C. Abbar, G. Journel, and A. Moise, C. R. Acad. Sci. Paris 5047, 365 (1965).

[4] J. Burie, J.-L. Destombes, A. Dubrulle, G. Journel, and C. Marliere, C. R. Acad. Sci. Paris 271, 1197 (1970).

[5] A. Dubrulle and J.-L. Destombes, C. R. Acad. Sci. Paris 274 B, 181 (1972).

[6] A. Dubrulle, These Science Physique, University of Lille 1972.

[7] A. Dubrulle and D. Boucher, C. R. Acad. Sci. Paris 278 B, 211 (1974).

[8] H. Dreizler, Mol. Phys. 59, 1 (1986).

[9] I. Merke, Dissertation, University of Kiel 1992.

[10] G. Bestmann, H. Dreizler, E. Fliege, and W. Stahl, J. Mol. Struct. 97, 215 (1983).

[11] G. Bestmann and H. Dreizler, Z. Naturforsch. 37 a, 58 (1982). atom changes the field gradient tensor at the chlorine atoms remarkably from $\chi_{c c}\left({ }^{35} \mathrm{Cl}\right)=\chi_{y y}\left({ }^{35} \mathrm{Cl}\right)=$ 47.7930(76) MHz, $\chi_{z z}\left({ }^{35} \mathrm{Cl}\right)=-78.58(25) \mathrm{MHz}, \eta=$ $\left(\chi_{x x}-\chi_{y y}\right) / \chi_{z z}=0.216(4)$ for $\mathrm{S}^{35} \mathrm{Cl}_{2}[18]$ and $\chi_{c c}\left({ }^{35} \mathrm{Cl}\right)$ $=\chi_{y y}\left({ }^{35} \mathrm{Cl}\right)=40.3560(94) \mathrm{MHz}, \chi_{z z}\left({ }^{35} \mathrm{Cl}\right)=-74.40(60)$ $\mathrm{MHz}, \eta=0.085$ (9) for $\mathrm{SO}_{2}{ }^{35} \mathrm{Cl}_{2}$.

For both molecules $\mathrm{SCl}_{2}$ and $\mathrm{SO}_{2} \mathrm{Cl}_{2}$ the $z$ axis of the coupling tensor is tilted out of the $\mathrm{S}-\mathrm{Cl}$ bond axis in the same direction and order of magnitude, $1.3^{\circ}$ in $\mathrm{SCl}_{2}$ and $2.0^{\circ}$ in $\mathrm{SO}_{2} \mathrm{Cl}_{2}$.

The ratio $q=Q\left({ }^{35} \mathrm{Cl}\right) / Q\left({ }^{37} \mathrm{Cl}\right)$ were calculated from $\chi_{y y}\left({ }^{35} \mathrm{Cl}\right) / \chi_{y y}\left({ }^{37} \mathrm{Cl}\right)$ avoiding an influence of the lower accuracy of $\chi_{a b}$. We obtained $q=1.26849$ (40) for $\mathrm{SCl}_{2}$ and $q=1.2677$ (89) for $\mathrm{SO}_{2} \mathrm{Cl}_{2}$. Both values agree within the error limits with $q=1.26878$ (15) [20].

\section{Acknowledgement}

We thank the members of our group for help and discussions. Funds of the Deutsche Forschungsgemeinschaft, Fonds der Chemie, and the Land Schleswig-Holstein are gratefully acknowledged. The calculations were made at the computer center of the University of Kiel.

[12] W. Stahl, G. Bestmann, H. Dreizler, U. Andresen, and R. Schwarz, Rev. Sci. Instrum. 56, 1759 (1985).

[13] W. Stahl, J. Gripp, N. Heineking, and H. Dreizler, Z. Naturforsch. 42a, 392 (1987).

[14] I. Merke and H. Dreizler, Z. Naturforsch. 43a, 196 (1988).

[15] J. Haekel and H. Mäder, Z. Naturforsch. 43a, 203 (1988).

[16] I. Merke, Chr. Keussen, and H. Dreizler, Z. Naturforsch. 45 a, 1273 (1990).

[17] W. Gordy and R. L. Cook, Microwave Molecular Spectra, John Wiley \& Sons, New York 1984, Chapter XV.

[18] I. Merke and H. Dreizler, Z. Naturforsch. 47 a, 1141 (1992).

[19] I. Merke and H. Dreizler, Z. Naturforsch. 47a, 1150 (1992).

[20] I.c. [16], App. E, 861. 